\title{
Preliminary characterization of metal-packaged fiber Bragg gratings under fatigue loading
}

\author{
Grzegorz Fusiek $^{\mathrm{a}}$, Tim Rubert ${ }^{\mathrm{a}}$, Paweł Niewczas ${ }^{\mathrm{a}}$, Jack McAlorum ${ }^{\mathrm{a}}$, Marcus Perry ${ }^{\mathrm{b}}$ \\ ${ }^{a}$ Electronic \& Electrical Engineering Department, University of Strathclyde, Glasgow, United Kingdom \\ ${ }^{\mathrm{b} C i v i l} \&$ Environmental Engineering Department, University of Strathclyde, Glasgow, United Kingdom \\ g.fusiek@strath.ac.uk
}

\begin{abstract}
This paper presents preliminary results for metalpackaged fiber Bragg grating strain and temperature sensors designed specifically for structural health monitoring in civil engineering applications. The laboratory experiments show that the in-house manufactured metal-packaged sensors are sufficiently resilient, under dynamic loading, to successfully undergo a million cycle fatigue test without adverse deterioration in performance. In addition, from the thermal characterization of the devices, a conclusion can be drawn that the metalpackaged sensors offer superior performance over sensors assembled using epoxy bonding. These early results are very promising, inspiring confidence in the adopted methodologies, and giving the mandate to proceed with more detailed laboratory testing to evaluate reliability and lifetime of the transducers in the future work.
\end{abstract}

Keywords-optical strain sensors, optical temperature sensors, fiber Bragg grating sensors, metallic encapsulation, fatigue loading.

\section{INTRODUCTION}

Fiber Bragg grating (FBG) sensors have been utilized for Structural Health Monitoring (SHM) applications in a wide range of industrial sectors such as aerospace [1]-[3], civil and environmental engineering [4]-[6], and nuclear engineering [7]-[9]. Since the sensors must monitor structural integrity and ensure long-term reliability even in adverse environmental conditions, appropriate sensor packaging and attachment are crucial for their operation and lifetime [4].

As an alternative to the existing FBG bonding and attachment methods utilizing epoxy or electroplating [10], [11], we previously developed a metal coated fiber sealing and bonding technique based on induction heating [12].

We also demonstrated experimentally that by selecting appropriate materials, such as kovar, brazing standard FBGs into metallic ferrules can be possible without any significant deterioration in the grating reflection [13]. Our recent work related to the high stress monitoring of prestressing tendons employing the developed technology demonstrated an improved performance over the epoxy or electroplating-based strain monitoring systems [14], [15].

Although much research focused on the application and implementation of such metal-packaged sensors, at present no analysis has been carried out to evaluate the sensors characteristics under fatigue loading.

In this paper, the sensors response to changing temperature and mechanical loading is presented. The experimental results indicate that all-metallic FBG encapsulation has the potential to offer a reliable solution for dynamically changing load and temperature monitoring in civil engineering applications.

\section{SENSORS DESIGN AND FABRICATION}

\section{A. Fiber Bragg gratings}

FBG sensors are formed as a result of UV-light inscribed periodic modulation of the refractive index along an optical fiber core over a length of $5-20 \mathrm{~mm}$. When such a structure is illuminated by a broadband infrared light, it reflects a relatively narrow part of the incident light spectrum with a distinctive peak in the light intensity around so called Bragg wavelength, $\lambda_{B}$. The spectral position of the peak is determined during the sensor fabrication process and described by the following relation:

$$
\lambda_{B}=2 n_{e f f} \Lambda
$$

where $n_{\text {eff }}$ is the effective refractive index of the FBG section and $\Lambda$ is the grating period. Any relative change in the grating period due to axial elongation or shortening of the fiber causes spectral shift of the FBG peak. Since the refractive index of the fiber is temperature dependent, the peak wavelength is a function of temperature.

A relative change in the FBG peak wavelength, $\Delta \lambda_{B} / \lambda_{B}$, due to a change in strain, $\Delta \varepsilon$, and temperature, $\Delta T$, can be expressed by

$$
\frac{\Delta \lambda_{B}}{\lambda_{B}}=C_{\varepsilon} \cdot \Delta \varepsilon+C_{T} \cdot \Delta T
$$

where $C_{\varepsilon}$ and $C_{T}$ are the strain and temperature sensitivities.

Clearly, to measure mechanical strain with an FBG, the sensor requires temperature compensation which is often realized by using an additional FBG temperature sensor decoupled from mechanical strain.

\section{B. Strain sensors}

To fabricate a strain sensor, a standard 10-mm long FBG inscribed in a 15-mm bare section of a single-mode copper coated fiber was encapsulated in a $25 \mathrm{~mm}$ long kovar capillary (outer diameter $0.7 \mathrm{~mm}$ ). The components were brazed together using a silver-based brazing paste and an induction heating system [12]. As the melting temperature of the brazing paste was around $620^{\circ} \mathrm{C}$, a stainless steel susceptor was used 
to apply heat directly at the joint location and to protect the FBG from excessive heat and deterioration [12], [13]. The assembly was then cooled down naturally to room temperature. Following the FBG encapsulation process, the sensors were equipped in additional metal shims to allow attachment to a weldable structure such as a reinforcement bar (rebar).

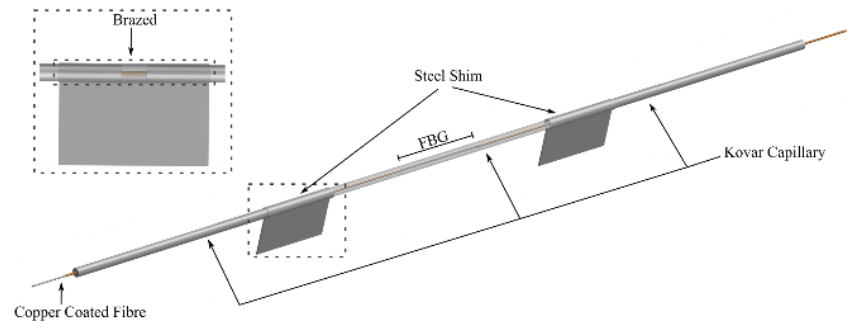

Figure 1. FBG-based strain sensor.

Two concurrent sensor variants for measuring strain were considered in this study, sharing the same geometry as illustrated in Figure 1, namely a prestressed and a nonprestressed strain sensor. The prestressed sensor involved brazing one end of a capillary to the metal coated fiber with an inscribed FBG and then prestressing the fiber prior to sealing the other end of capillary. This process introduced an additional positive offset in the FBG peak wavelength, allowing for increasing the sensor operation range in the compression mode. In the non-prestressed design, the FBG remained prestress free.

\section{Temperature sensors}

Three temperature sensor constructions were evaluated in this study: a metal-packaged temperature sensor incorporating a brazed kovar capillary and steel shim (Figure 2a), an epoxy bonded design (Figure 2b), and a brazed kovar capillary and soldered copper tube and shim (Figure 2c).

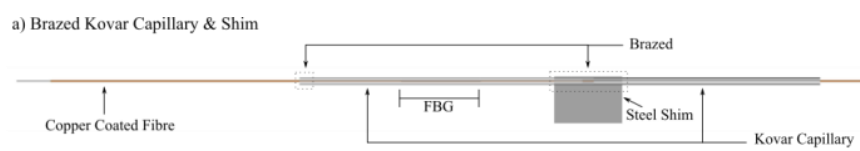

b) 2 Epoxy Bonded Copper Tubes

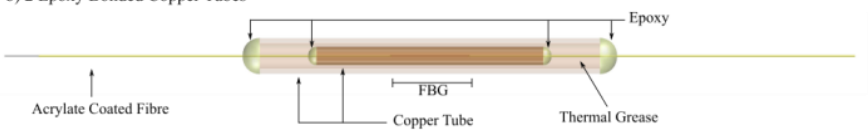

c) Brazed Kovar Capillary and Soldered Copper Tube \& Shim

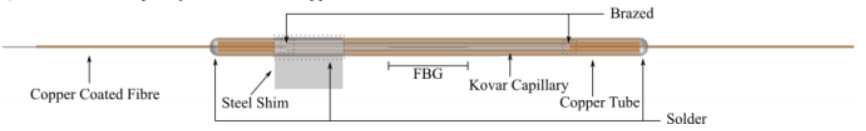

Figure 2. Temperature sensor constructions.

The brazed kovar capillary and steel shim temperature sensor was fabricated in the same way as the non-prestressed strain sensor described in the previous section. A steel shim was brazed to one end of the capillary only to prevent the mechanical strains being transferred to the sensor. Two remaining temperature sensor constructions involved encapsulating the temperature sensors into additional copper tubes providing additional isolation from external strains. The epoxy based temperature sensor consisted of two copper tubes that were sealed with epoxy. The copper tube in the third design, shown in Figure 2c, was soldered to the fiber using a low temperature solder with melting point below $300{ }^{\circ} \mathrm{C}$. The lengths of the temperature sensors ranged between 20 and 32 $\mathrm{mm}$.

\section{THERMAL CHARACTERISATION AND CALIBRATION}

To estimate thermal sensitivities of the strain and temperature sensors and to allow for temperature compensation of the strain sensors [16], the devices were thermally characterized before and after their attachment to a steel rebar (B500B; ø20 mm).

The response of the metal-packaged and epoxy-based temperature sensors when thermally cycled between $25^{\circ} \mathrm{C}$ and $65{ }^{\circ} \mathrm{C}$ are shown in Figure 3 and Figure 4. The metalpackaged sensor has good linearity and repeatability after three cycles while the epoxy based sensor response drifts significantly with every subsequent temperature cycle.

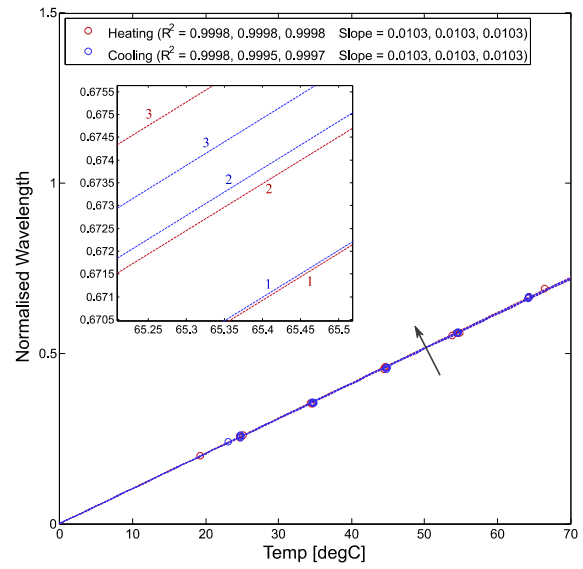

Figure 3. Temperature response of a metal-packaged temperature sensor thermally cycled three times.

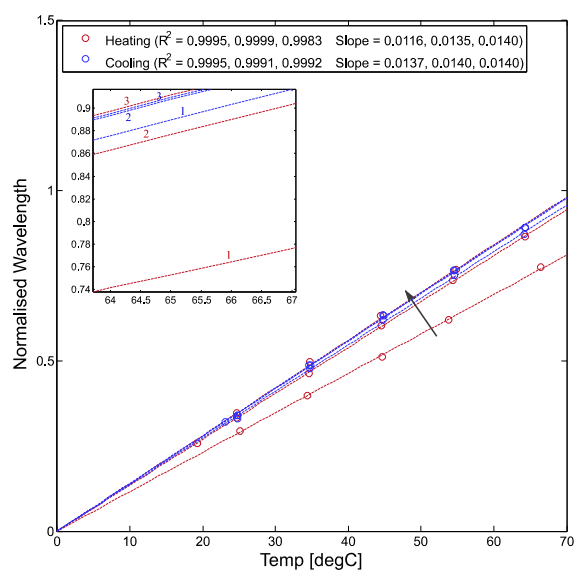

Figure 4. Temperature response of an epoxy-based temperature sensor thermally cycled three times.

The temperature characteristics of the prestressed and nonprestressed metal-packaged strain sensors when attached to a rebar are shown in Figure 5 and Figure 6. Both sensors show good linearity and repeatability during temperature cycling. The increase in the sensors thermal response sensitivity due to the influence of the rebar and its higher thermal expansion was observed. 
Since the epoxy based temperature sensor seemed inferior to the metal-packaged sensors, demonstrating drift, this type of sensor was not utilized for the fatigue tests.

TABLE I presents the results of the thermal calibration of both temperature sensors as well as the results of the two strain sensors respectively. The coefficients were used for temperature compensation of the strain sensors.

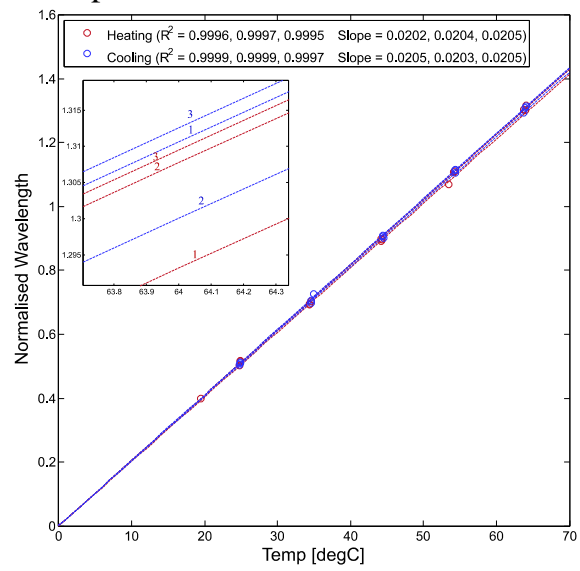

Figure 5. Temperature response of a metal-packaged prestressed strain sensor thermally cycled three times.

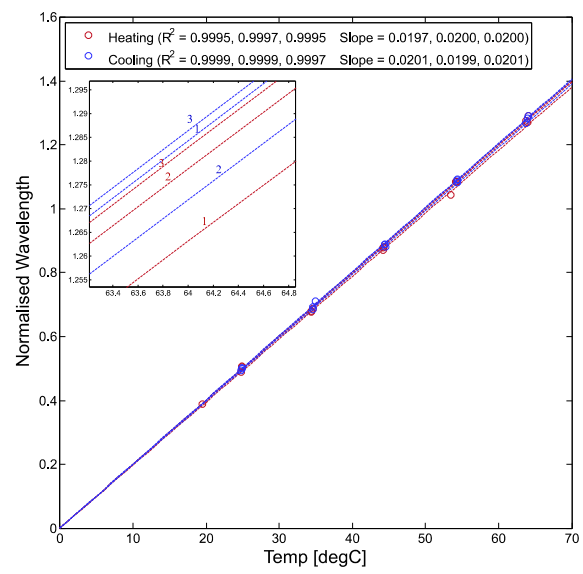

Figure 6. Temperature response of a metal-packaged nonprestressed strain sensor thermally cycled three times.

\section{FATIGUE EXPERIMENT AND RESULTS}

The reinforcement bar with the attached spot welded sensors was cycled under axial fatigue loading in an Instron 8802 servo-hydraulic machine as illustrated in Figure 7.

The machine force was set to vary sinusoidally in the range of $\pm 31.4 \mathrm{kN}$ (stress ratio $(\mathrm{R})=-1$; fully reversed), corresponding to $\pm 100 \mathrm{MPa}$ of stress or $\pm 0.5 \mathrm{~m} \varepsilon$ of strain at a frequency of $10 \mathrm{~Hz}$. The machine data (force and displacement) was recorded with a frequency of $1 \mathrm{kHz}$, whereas the sensors were interrogated with a commercial interrogator at a frequency of $250 \mathrm{~Hz}$, resulting in 25 points per period. After completion of $10^{6}$ cycles the test was stopped.

Figure 8 depicts readings from both prestressed and nonprestressed sensors that were analyzed using a fast Fourier transform (FFT), where the peak magnitude at $10 \mathrm{~Hz}$ was re- transformed into the amplitude (time domain) and plotted for one million cycles. The inset in Figure 8 shows the machine force and non-prestressed strain sensor data normalized with respect to its maximum value for the considered length of 23 periods, cross-correlated in Matlab, and then plotted on the same graph. This illustrates how the actual sensor output agrees with the machine input. The sensor appears to represent the force amplitude and phase faithfully.
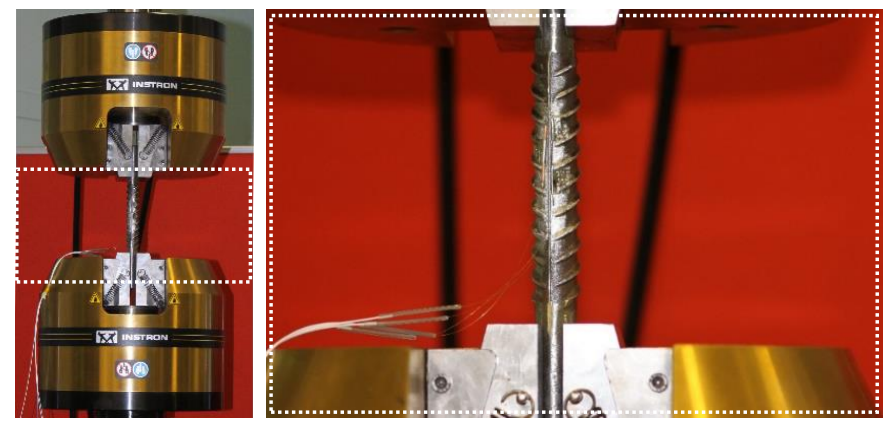

Figure 7. Sensors under fatigue testing.

From the fatigue test results, the non-prestressed sensor output reduction can be observed within the first 70,000 cycles, decreasing from an initial amplitude of $0.39 \mathrm{~nm}$ down to $0.185 \mathrm{~nm}$. The prestressed sensor appears to behave fairly consistently with an initial amplitude response of $0.31 \mathrm{~nm}$ reducing to $0.28 \mathrm{~nm}$ until reaching $2 * 10^{5}$ cycles and then remaining constant over the lifetime of $10^{6}$ cycles until the test was stopped. Upon visual inspection, the spot welding deterioration was observed in both cases causing partial disattachment of the sensors from the rebar, making impossible to maintain a constant strain transfer to the sensor. This observation was much more prominent for the nonprestressed strain sensor than the prestressed strain sensor. Nevertheless, both sensors survived successfully a one million cycles fatigue test without damage of the metal package.

Concerning the temperature sensors responses to dynamic loading, both designs isolated the reinforcement bar mechanical strain.

\section{CONCLUSIONS}

This work has presented early research on fatigue evaluation of metal-packed strain and temperature sensors when attached to a B500B reinforcement bar under a fully reversed load cycle of $\pm 0.5 \mathrm{~m} \varepsilon$ with a total of $10^{6}$ cycles. The results revealed that the spot welding quality was not sufficient in this case to maintain constant strain transfer during the test. The brazed capillary with soldered copper tube and shim is the preferred construction for the temperature sensors. Future work will focus on improving the spot welding quality and performing more detailed laboratory testing to further evaluate reliability and lifetime of the sensors.

\section{ACKNOWLEDGEMENTS}

Research presented in this paper was funded by the industrial partners SSE and SPR, and the EPSRC, grant number EP/L016680/1. 
TABLE I. RESULTS FROM THE THERMAL CHARACTERISATION

\begin{tabular}{cccccccc}
\hline \multirow{2}{*}{ Sensor } & \multicolumn{3}{c}{ Senstivity $\left[\mathrm{pm} /{ }^{\circ} \mathrm{C}\right]$} & \multicolumn{4}{c}{ Linear Fit $\left(R^{2}\right)$} \\
& $\mathrm{C} 1$ & $\mathrm{C} 2$ & $\mathrm{C} 3$ & $\mathrm{C} 1$ & $\mathrm{C} 2$ & $\mathrm{C} 3$ \\
\hline Brazed Kovar Capillary and Shim & 17.4 & 17.3 & 17.4 & 0.9996 & 0.9998 & 0.9996 \\
Brazed Kovar Capillary with Copper Tube \& Soldered Shim & 14.4 & 14.5 & 14.6 & 1 & & 0.9999 \\
\hline \hline Prestressed Strain Sensor & 20.3 & 20.4 & 20.5 & 0.9998 & 0.9998 & 0.9996 \\
Non-prestressed Strain Sensor & 19.9 & 20 & 20 & 0.9997 & 0.9998 & 0.9996 \\
\hline
\end{tabular}

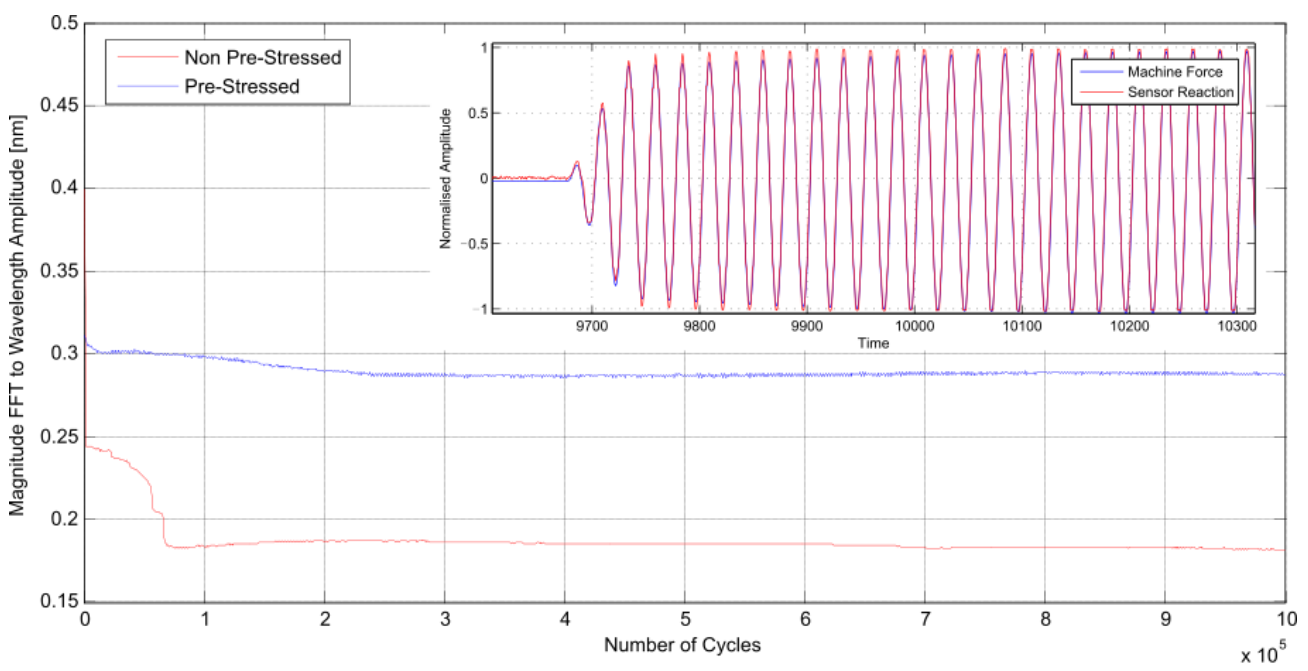

Figure 8. Fatigue test results.

\section{REFERENCES}

[1] Li, J., Zhou, Z., and Ou, J., (2004b). "Sensors and smart structures technologies for civil, mechanical, and aerospace systems." Proc., SPIE, San Diego, 5765(2), SPIE, Bellingham, Wash., 265-274

[2] G. C. Kahandawa, J. Epaarachchi, H. Wang, and K. T. Lau, "Use of FBG sensors for SHM in aerospace structures," Photonic Sensors, vol. 2, no. 3, pp. 203-214, Jul. 2012.

[3] A. Panopoulou, D. Roulias, T. H. Loutas, and V. Kostopoulos, "Health Monitoring of Aerospace Structures Using Fibre Bragg Gratings Combined with Advanced Signal Processing and Pattern Recognition Techniques," Strain, vol. 48, no. 3, pp. 267-277, Jun. 2012

[4] Li, H.N., Li, D.S. and Song, G.B. "Recent applications of fiber optic sensors to health monitoring in civil engineering", Engineering Structures, 26(11), 1647-1657 (2004).

[5] T.H.T. Chan, L. Yu, H.Y. Tam, Y.Q. Ni, S.Y. Liu. W.H. Chung, L.K. Cheng, "Fiber Bragg grating sensors for structural health monitoring of Tsing Ma bridge: Background and experimental observation", Engineering Structures, vol. 26, no. 5, pp. 648-659, April 2006.

[6] Daniele Inaudi, Branko Glisic, "Application of distributed Fiber Optic Sensory for SHM", $2^{\text {nd }}$ International Conference on Structural Health Monitoring of Intelligent Infrastructure, 2005.

[7] J. R. Lee, S. Y. Chong, C. Y. Yun, and H. Sohn, "Design of Fiber Bragg Grating Acoustic Sensor for Structural Health Monitoring of Nuclear Power Plant,” Adv. Mater. Res., vol. 123-125, pp. 859-862, Aug. 2010.

[8] P. Crolla, P. Niewczas, \& J. Mcdonald, 'An alternative method for the monitoring of strain and temperature in a nuclear fusion reactor', $44^{\text {th }}$ International Universities' Power Engineering Conference, Glasgow, United Kingdom, (2009)
[9] P. Niewczas, G. Fusiek, \& P. Crolla, 'Concept level evaluation of the full-scale deployment of fibre bragg grating sensors for measuring forces in JET during plasma disruption events', $5^{\text {th }}$ International Conference on Condition Monitoring and Machine Failure Prevention Technologies, Edinburgh, UK, (2008)

[10] Li, E., Xi, J., Chicharo, J.F., Liu, T., Li, X., Jiang, J., Li, L., Wang, Y., Zhang, Y., "The Experimental evaluation of FBG sensor for strain measurement of prestressed steel strand," Proc. SPIE Smart Structures Devices and Systems II 5649, 463-469 (2004)

[11] Yun Tu and Shan-Tung Tu, "Fabrication and characterization of a metal-packaged regenerated fiber Bragg grating strain sensor for structural integrity monitoring of high-temperature components", Smart Mater. Struct. 23(3) (2013)

[12] P. Niewczas and G. Fusiek, "Induction heating assisted optical fiber bonding and sealing technique," in 21st International Conference on Optical Fiber Sensors, 2011, p. 77536H.

[13] Perry, M., Niewczas, P., Johnston, M., Cook, K. and Canning J., "Induction Brazing of Type-I Fiber Bragg Gratings Into Kovar Ferrules Exploiting Curie Transition”, IEEE Sensors 13(2), 816-823 (2013).

[14] M. Perry, Z. Yan, Z. Sun, L. Zhang, P. Niewczas, M. Johnston, "High stress monitoring of prestressing tendons in nuclear concrete vessels using fibre-optic sensors," Nuclear Engineering and Design 268, 35-40 (2014)

[15] G. Fusiek, M. Perry, and M. Johnston, "Deformation monitoring in prestessing tendons using fibre Bragg gratings encapsulated in metallic packages," 23rd Int. Conf. Opt. Fibre Sensors, vol. 9157, pp. 14-17, 2014.

[16] I. Mckeeman, G. Fusiek, M. Perry, P. Niewczas, and M. Johnston, "Insitu temperature calibration procedure for temperature and strain fibre Bragg grating sensors for monitoring pre-stressing strands," vol. 9634, no. 1 , p. $96345 \mathrm{I}, 2015$ 